\title{
Toxic effect of using twenty percent alcohol on corneal epithelial tight junctions during LASEK
}

\author{
PENG ZHANG ${ }^{1,2^{*}}$, MIN LIU $^{2 *}$ and RONGFENG LIAO ${ }^{1}$ \\ ${ }^{1}$ Department of Ophthalmology, The First Hospital Affiliated of Anhui Medical University, Hefei, Anhui 230022; \\ ${ }^{2}$ Department of Ophthalmology, the Fourth Affiliated Hospital of Anhui Medical University, Hefei, Anhui 230022, P.R.China
}

Received December 8, 2011; Accepted March 23, 2012

DOI: $10.3892 / \mathrm{mmr} .2012 .880$

\begin{abstract}
The aim of this study was to determine the effect of using $20 \%$ alcohol on corneal epithelial tight junctions during laser epithelial keratomileusis (LASEK). Sixty SpragueDawley rats were randomly divided into two equal groups. The central area of the rat corneas in one group were demarcated with a 3-mm trephine, treated with $20 \%$ alcohol for $45 \mathrm{sec}$ and washed with sterile balanced salt solution. The epithelium was removed by an epithelial microhoe used in LASEK. In the other group, the rat corneal epithelium in the central area was mechanically scraped. The experimental animals were sacrificed at 24, 48, 72, 96 and $120 \mathrm{~h}$ after surgery. The levels of the tight junction proteins, claudin-1 and ZO-1, were determined by immunofluorescence and semi-quantitative reverse transcription-polymerase chain reaction (RT-PCR) analyses. We found that at approximately $48 \mathrm{~h}$ after surgery, the wounded corneas were replaced by corneals with regenerated epithelium. Immunofluorescence analysis demonstrated that the expressions of claudin-1 and ZO-1 in the corneal epithelium of the alcohol-treated group were weaker compared to the mechanical group at the 24 and $48 \mathrm{~h}$ time-points. Semi-quantitative RT-PCR analysis showed that the mRNA levels of claudin-1 and ZO-1 in the central cornea after alcohol treatment were lower compared to those in the mechanical group from 24 to $48 \mathrm{~h}$, with no significant difference after $72 \mathrm{~h}$. Thus, we conclude that the treatment with $20 \%$ alcohol during LASEK results in damage to the corneal epitheleal tight junctions and prolongs normal recovery time.
\end{abstract}

\section{Introduction}

In the eye, the cornea is the most refractive part and even a slight change in its curvature can have a significant effect on its

Correspondence to: Dr RongFeng Liao, Department of Ophthalmology, The First Affiliated Hospital of Anhui Medical University, 218 Jixi Road, Hefei, Anhui 230022, P.R. China

E-mail: liaorfayfy@yahoo.com.cn

*Contributed equally

Key words: laser-assisted subepithelial keratectomy, cornea, tight junction refractive status. Since Jose Barraquer recognized this fact and was the first to introduce lamellar refractive surgery techniques, many techniques have been developed, such as photorefractive keratectomy (PRK), laser in situ keratomileusis (LASIK) and laser epithelial keratomileusis (LASEK), all of which involve the removal of corneal tissue (1). LASEK was performed by Azar et al in 1996, which they termed alcohol-assisted flap PRK (2). This procedure was later named by Camellin (3). It was conceived as a hybrid between PRK and LASIK. LASEK is more comfortable and less traumatic for patients with deep set eyes or narrow palpebral apertures, where the application of a laser keratome may be more difficult. Recent results have shown that LASEK is an effective option, a safe and successful procedure; however, the LASEK procedure involves chemically reducing the corneal epithelium adhesion to the underlying Bowman's layer using a diluted solution of alcohol, which is potentially toxic to the epithelium and corneal stroma (1). Although after treatment with alcohol, the cornea is washed using cold-balanced salt solution, the toxic effects of alcohol on the cornea cannot be neglected. It has been reported that in the early post-operative period, transient corneal epithelial erosion is common after LASEK and is possibly related to the use of alcohol.

The corneal epithelium functions as the first line of defense. The intact epithelium not only protects the eye from the outside environment, but also regulates the passive movement of fluid, electrolytes, macromolecules and cells through the paracellular pathway. Tight junctions are present at the apical side of epithelia and are thought to play a significant role in the establishment and maintenance of this important defense for the eye $(4,5)$. According to existing studies, the tight junction complex consist of three groups of proteins: transmembrane proteins (claudin, occludin and junctional adhesion molecules), peripheral membrane proteins (ZO-1, ZO-2, ZO-3 and MUPP-1), which have PDZ domains and bind to transmembrane proteins, and cytoplasmic proteins (cingulin and 7H6 antigen), which exist around tight junctions without any direct binding (6).

Claudin, which was first discovered in 1998, comprises a family of transmembrane proteins and 24 types have been identified to date (6). Although claudin molecules also bear four transmembrane domains, there is no sequence similarity between claudins and occludin. Different mixture of claudins and occludin create tight junction strands that are associated laterally with strands of adjacent cells, forming paired strands 
that eliminate extracellular space (7). ZO-1, ZO-2 and ZO-3 are members of the membrane-associated guanylate kinase homologue (MAGUK) family, which is distinguished by a core cassette of protein-binding domains. The MAGUK proteins are able to interact with each other, with occludin and claudins, and with the actin cytoskeleton to form tight junction complexes (8).

In this study, we examined the distribution and restoration of the tight junction proteins, claudin- 1 and ZO-1, in rat corneal epithelium treated with $20 \%$ alcohol to investigate the toxic effects of using $20 \%$ alcohol during LASEK on corneal epithelial tight junctions.

\section{Materials and methods}

Animals. Sixty-two Sprague-Dawley rats weighing 250-350 g were used in this study. The experiment conformed to the guidelines set out in the Association for Research in Vision and Ophthalmology Statement for Use of Animals in Ophthalmic and Vision Research.

Surgical procedure. Sixty rats were randomly divided into two equal groups and anesthetized with $3 \%$ pentobarbital sodium $(1 \mathrm{mg} / \mathrm{kg})$, followed by topical anesthesia of the anterior surface of the eye with $0.1 \%$ Alcaine eye drops. The central area of the rat corneas in one group were demarcated with a 3-mm trephine, treated with $20 \%$ alcohol for $45 \mathrm{sec}$, then washed with sterile balanced salt solution. The epithelium was removed by an epithelial microhoe used in LASEK. In the other group, rat corneal epithelium in the central area was mechanically scraped by a blunt spatula. After surgery, $3 \%$ levofloxacin eye drops were applied three times per day. Two rats which underwent no eye surgery were used as the blank controls. Experimental animals were sacrificed at 24, 48, 72 , 96 and $120 \mathrm{~h}$ after surgery.

Histological examination. Rat eyes were embedded in OCT medium and frozen for cryosectioning. Sections $(6 \mu \mathrm{m})$ were cut and stained with H\&E for histological examination.

Immunfluorescence. After three washings with PBS for $5 \mathrm{~min}$, the sections were blocked by $5 \%$ non-fat milk for $2 \mathrm{~h}$ at $37^{\circ} \mathrm{C}$, washed with PBS three times for 5 min again, and the primary antibodies to claudin-1 (Santa Cruz; 1:200 dilution) and ZO-1 (Invitrogen; 1:100 dilution) were applied overnight at $4^{\circ} \mathrm{C}$. After incubation with the primary antibody, the sections were washed three times with PBS. The fluorescein isothiocyanate (FITC)-conjugated secondary antibodies were then applied to the sections followed by incubation for $1 \mathrm{~h}$ at room temperature. After mounting, sections were photographed under a microscope (Eclipse 80i; Nikon, Tokyo, Japan) equipped with a digital camera.

Semi-quantitative reverse transcription-polymerase chain reaction (RT-PCR). For semi-quantitative RT-PCR, total RNA was isolated from the central region (diameter, $3 \mathrm{~mm}$ ) of the experimental cornea with the use of TRIzol (Invitrogen). The concentration of RNA was estimated by spectrophotometry using UV absorbance at 260 and $280 \mathrm{~nm}$. Denaturing agarose gel electrophoresis was used to determine the integrity of the RNA. Portions $(2 \mu \mathrm{g})$ of the RNA were subjected to RT-PCR analysis with a RT-PCR kit (Promega). The PCR protocol was designed to maintain amplification in the exponential phase. The primer sequences (forward and reverse, respectively) were 5'-GGCCACYSTTGGMATGAAGT-3' and 5'-GCCARTGAA RAGAGCCTGAC-3' for claudin-1; 5'-GHGGCATWTTT AACAGCAAT-3' and 5'-GKAGCTCCACRGGCTTCAGG-3' for ZO-1; and 5'-TGCCGAAGTGGTCGTGGATGACCT-3' and 5'-GCGCCTGGTCACCAGGGCTGCTT-3' for GAPDH. RT was carried out at $42^{\circ} \mathrm{C}$ for $60 \mathrm{~min}$, then at $72^{\circ} \mathrm{C}$ for $15 \mathrm{~min}$, followed by an ice bath for $2 \mathrm{~min}$. PCR amplification comprised of incubations for 30 cycles at $94^{\circ} \mathrm{C}$ for $30 \mathrm{sec}, 57^{\circ} \mathrm{C}$ (claudin1), $58^{\circ} \mathrm{C}$ (ZO-1 and GAPDH) for $30 \mathrm{sec}$ and $72^{\circ} \mathrm{C}$ for $30 \mathrm{sec}$. Samples were finished with a final extension at $72^{\circ} \mathrm{C}$ for $5 \mathrm{~min}$ and cooled to $4^{\circ} \mathrm{C}$. Finally, the amplification products were fractionated by electrophoresis on a $1.5 \%$ agarose gel and stained with ethidium bromide.

Statistical analysis. Quantitative data are reported as the means \pm SD and were analyzed by the independent-samples t-test and two-way analysis of variance using SPSS statistical software (version 15.0). A P-value of $<0.05$ was considered to indicate a statistically significant difference.

\section{Results}

Histological examination. The examination of morphological changes in the corneal epithelium showed that the epithelium was stratified (2-3 cell layers) at $24 \mathrm{~h}$ after surgery and that epithelial cells had recovered from all wounds (treated with either alcohol or mechanical procedure) at $48 \mathrm{~h}$ post-wounding. There was no significant difference between the two groups during epithelial wound closure (Fig. 1).

Immunfluorescence. In the unwounded cornea, ZO-1 was confined to the superficial cell layer of the corneal epithelium. At $24 \mathrm{~h}$ after alcohol treatment, compared to the mechanical group, weaker and fainter ZO-1 staining was observed at the wounded area. At $48 \mathrm{~h}$, the ZO-1 expression in the cornea treated with alcohol appeared to become more organized; however, it was still weaker than that of the cornea treated mechanically. At $72 \mathrm{~h}$, there was no significant change between the two groups and the ZO-1 staining was consistent with the unwounded corneal epithelium at 96 and $120 \mathrm{~h}$ after surgery in both groups (Fig. 2).

Claudin-1 in the unwounded cornea was localized at all the cell layers of the corneal epithelium. At $24 \mathrm{~h}$ after alcohol treatment, the pattern of claudin-1 localization in the alchoholtreated corneas was more faint and disorganized than that of the corneas treated mechanically. At $48 \mathrm{~h}$ post-surgery, this faint disorganized pattern of claudin-1 in the cornea which was treated by alcohol appeared to become more organized and stronger compared to the pattern at $24 \mathrm{~h}$; however, it was still faint and weak. At $72 \mathrm{~h}$, claudin-1 staining in both groups was more organized and was approaching the pattern of localization observed in the unwounded cornea. At 96 and $120 \mathrm{~h}$ post-surgery, there was no significant difference in claudin-1 expression between the two groups (Fig. 3).

Semi-quantitative RT-PCR. The relative expression levels of claudin-1 and ZO-1 mRNA in the central cornea in the 

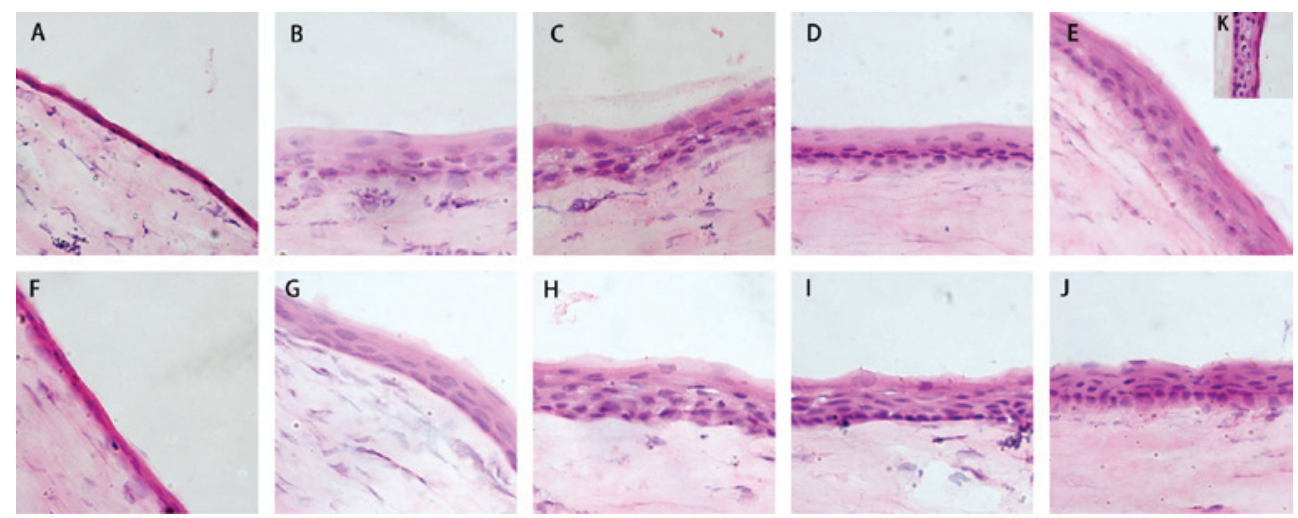

Figure 1. The examination of morphological changes in the corneal epithelium showed that (A and F) the epithelium was stratified (2-3 cell layers) at $24 \mathrm{~h}$ after surgery and (B and G) epithelial cells had recovered from all wounds (treated with either alcohol or the mechanical procedure) at $48 \mathrm{~h}$ post-wounding. There was no significant difference between the two groups at (C and H) 72, (D and I) 96 and (E and J) $120 \mathrm{~h}$ after surgery. (K) Normal cornea. Bar, $50 \mu \mathrm{m}$.
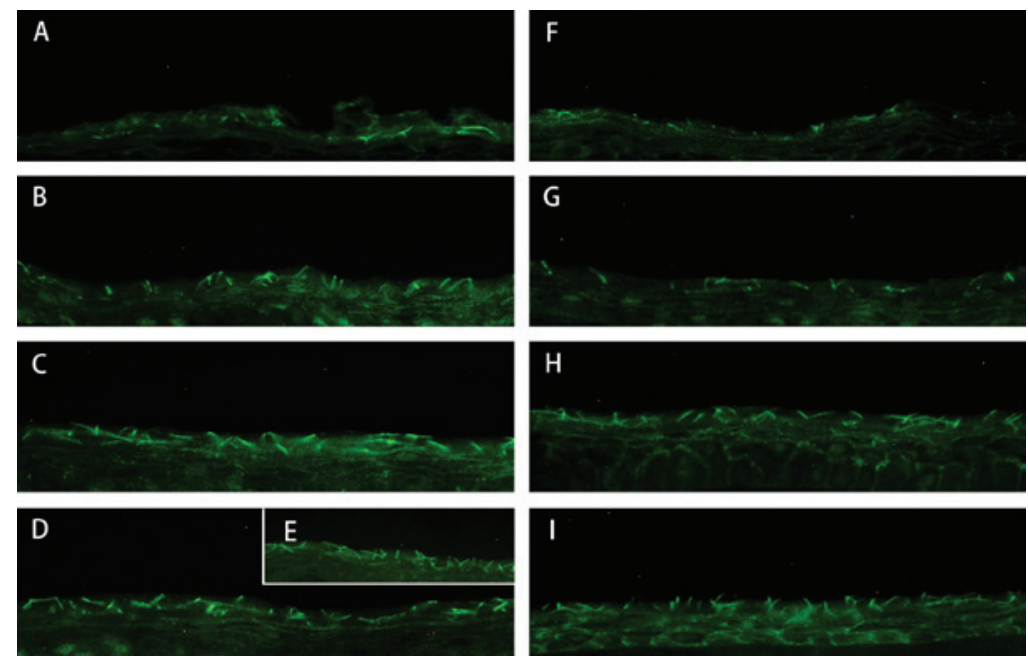

Figure 2. (E) In the unwounded cornea, ZO-1 was confined to the superficial cell layer of the corneal epithelium. At $24 \mathrm{~h}$ after alcohol treatment, compared to (A) the mechanical group, (F) weaker and fainter ZO-1 staining was observed at the wounded area. (G) At $48 \mathrm{~h}$, the ZO-1 expression in the cornea treated with alcohol appeared to become more organized; (B) however, it was still weaker than that of the cornea treated mechanically. At $72 \mathrm{~h}$, there was no significant change between the two groups $(\mathrm{C}$ and $\mathrm{H})$ and the ZO-1 staining was consistent with the unwounded corneal epithelium at (D and I) 96 and $120 \mathrm{~h}$ (data not shown) after surgery in both groups. Bar, $50 \mu \mathrm{m}$.
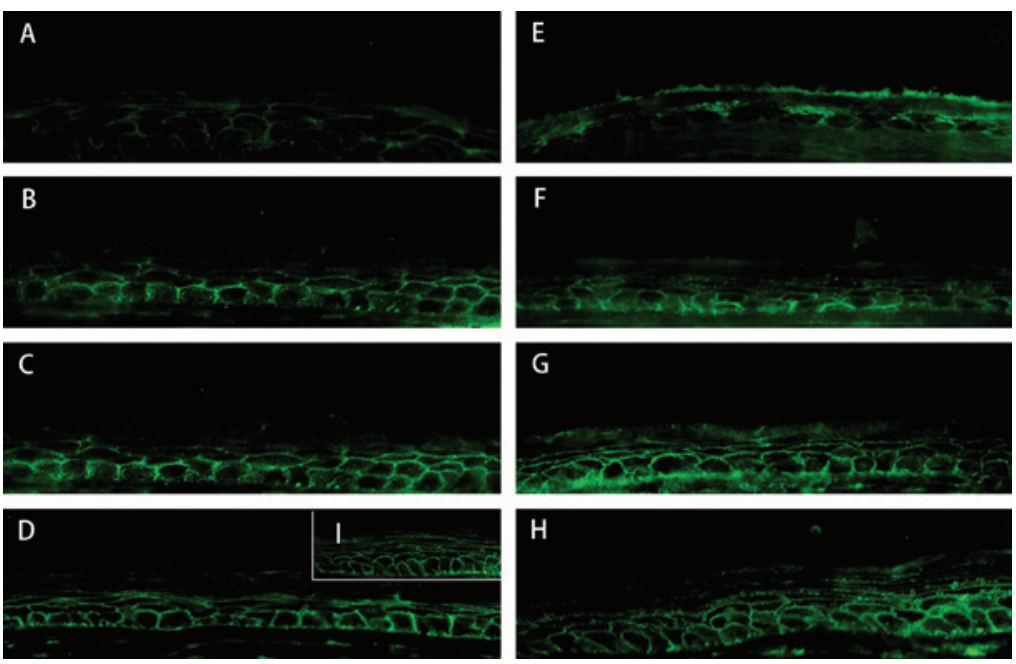

Figure 3. (I) Claudin-1 in the unwounded cornea was localized to all cell layers of the corneal epithelium. (E) At $24 \mathrm{~h}$ after alcohol treatment, the pattern of claudin-1 localization was more faint and disorganized than that of the cornea treated mechanically (A). (F) At $48 \mathrm{~h}$ post-surgery, this faint disorganized pattern of claudin-1 in the cornea treated with alcohol appeared to become more organized and stronger compared to the pattern at $24 \mathrm{~h}$; however, it was still faint and weaker than that of the cornea treated mechanically (B). At $72 \mathrm{~h}$, claudin-1 staining in both groups was more organized and was approaching the pattern of localization observed in the unwounded cornea $(\mathrm{C}$ and $\mathrm{G})$. At (D and $\mathrm{H}) 96$ and $120 \mathrm{~h}$ (data not shown) post-surgery, there was no significant difference in claudin-1 expression between the two groups and the intact cornea. Bar, $50 \mu \mathrm{m}$. 
Table I. Relative expression levels of claudin-1 mRNA to GAPDH.

\begin{tabular}{lccccc}
\hline Group & $24 \mathrm{~h}$ & $48 \mathrm{~h}$ & $72 \mathrm{~h}$ & $96 \mathrm{~h}$ & $120 \mathrm{~h}$ \\
\hline Alcohol treatment & $0.11 \pm 0.02$ & $0.18 \pm 0.02$ & $0.24 \pm 0.02$ & $0.27 \pm 0.04$ & $0.28 \pm 0.03$ \\
Mechanical treatment & $0.19 \pm 0.03^{\mathrm{a}}$ & $0.26 \pm 0.03^{\mathrm{a}}$ & $0.28 \pm 0.03$ & $0.29 \pm 0.02$ & $0.29 \pm 0.02$ \\
\hline
\end{tabular}

${ }^{\mathrm{a}} \mathrm{vs}$. the mechanically-treated group, $\mathrm{p}<0.05$.

Table II. Relative expression levels of ZO-1 mRNA to GAPDH.

\begin{tabular}{lccccc}
\hline Group & $24 \mathrm{~h}$ & $48 \mathrm{~h}$ & $72 \mathrm{~h}$ & $96 \mathrm{~h}$ & $120 \mathrm{~h}$ \\
\hline Alcohol treatment & $0.16 \pm 0.04$ & $0.28 \pm 0.04$ & $0.52 \pm 0.07$ & $0.59 \pm 0.05$ & $0.60 \pm 0.04$ \\
Mechanical treatment & $0.26 \pm 0.03^{\mathrm{a}}$ & $0.37 \pm 0.08^{\mathrm{a}}$ & $0.50 \pm 0.04$ & $0.58 \pm 0.05$ & $0.59 \pm 0.06$
\end{tabular}

${ }^{\mathrm{a}}$ VS. the mechanically-treated group, $\mathrm{p}<0.05$.

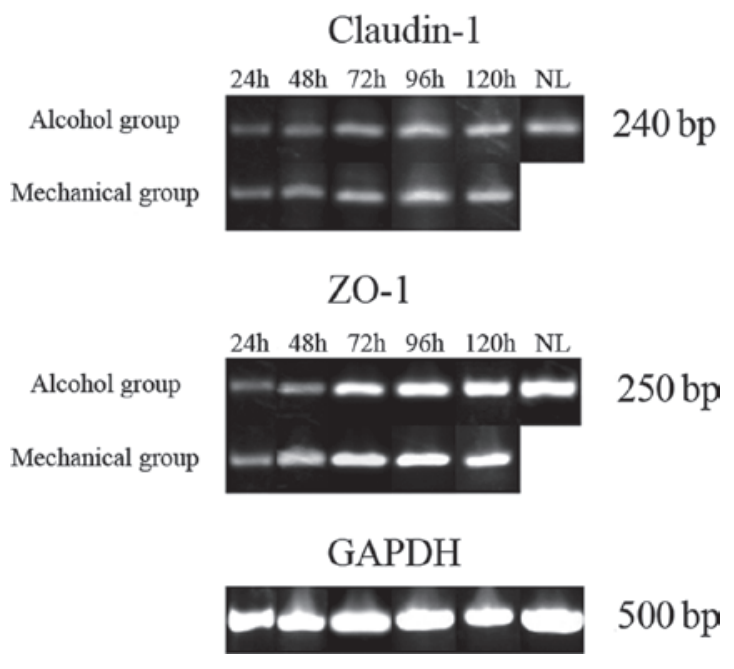

Figure 4. Gel electrophoresis of RT-PCR products of claudin-1 and ZO-1 after alcohol and mechanical treatment. NL, normal cornea.

group treated with alcohol and that treated mechanically are shown in Tables I and II, and Fig. 4. RT-PCR analysis demonstrated that the claudin-1 and ZO-1 mRNA levels 24 and $48 \mathrm{~h}$ after treatment with alcohol were significantly lower than those observed 24 and $48 \mathrm{~h}$ after the mechanical treatment $(\mathrm{P}<0.05)$, and the expression levels of $\mathrm{ZO}-1$ and claudin-1 mRNA between the two groups were the same at 72, 96 and $120 \mathrm{~h}(\mathrm{P}>0.05)$. We also used two-way analysis of variance to analyze the interaction between surgical methods and time after surgery (Tables III and IV). We found the existence of an interaction between surgical methods and time after surgery and, after both treatments, the mRNA expressions of claudin-1 and ZO-1 increased and recovery occurred as time went by.

\section{Discussion}

Alcohol has long been used as a treatment agent in the ophthalmologic field. At first, a retrobulbar injection of $90 \%$ alcohol was used in an attempt to relieve intractable ocular pain in patients with neovascular glaucoma (9). In the mid1990s, low concentrations of alcohol were used to remove the corneal epithelium in excimer laser refractive surgery. Due to its possible ocular toxicity, the effects of alcohol on the cornea have been re-evaluated. Campos et al (10) found that there was a significant decrease in rabbit cornea stromal keratocytes $24 \mathrm{~h}$ after using 100\% alcohol for $2 \mathrm{~min}$. Agrawal et al (11) reported an increased inflammatory response and damaging effects on keratocytes when using $70 \%$ isopropyl alcohol for 2 min for epithelium removal in rabbit corneas.

At present, 20\% alcohol is commonly used to create an epithelial flap during LASEK. Since the corneal epithelium is highly resistant to pathogen penetration, the epithelium tries to restore its protective barrier as quickly and efficiently as possible after surgery. However, the recovery of the wounded area does not necessarily mean that the epithelial barrier is restored. Numerous studies had examined the effects of various factors on wounding and when certain intercellular junctions re-appear. They showed that the restoration of the barrier lags beyond epithelial wound closure (12). Therefore, the rebuilding of the cell-cell junction is critical during epithelial wound healing after refractive surgery. The junctional complex of epithelial cells consists of three distinct components: tight junctions, adherence junctions and desmosomes. The tight junction plays an important role among the various types of intercellular junctions. It appears that the early restoration of the tight junction is crucial for preventing unwanted substances from the environment entering intercellular spaces.

According to previous studies, tight junctions have been detected in the corneal epithelium and most prominently in the superficial cell layer $(13,14)$. Different tight junction proteins are known to be targeted for barrier modulation. Claudins comprise a multigene family consisting of approximately 24 members, which form tight junction strands that are associated laterally with those of adjacent cells to form paired strands to eliminate the extracellular space. At the molecular level, distinct species of claudins interact with themselves and with each other, within and between tight junction strands (8). $\mathrm{ZO}-1$, which is expressed in superficial and sub-superficial 
Table III. Interaction between surgical methods and time after surgery of claudin-1.

\begin{tabular}{|c|c|c|c|c|c|}
\hline Items & Type III sum of squares & df & Mean square & $\mathrm{F}$ & Sig. \\
\hline Surgical methods & 0.032 & 1 & 0.032 & 39.629 & 0.000 \\
\hline Time after surgery & 0.105 & 4 & 0.026 & 32.386 & 0.000 \\
\hline Surgical methods - time after surgery & 0.015 & 4 & 0.004 & 4.482 & 0.004 \\
\hline
\end{tabular}

df, degree of freedom; F, F test results; Sig., significance.

Table IV. Interaction between surgical methods and time after surgery of ZO-1.

\begin{tabular}{lccccr}
\hline Items & Type III sum of squares & df & Mean square & F & Sig. \\
\hline Surgical methods & 0.013 & 1 & 0.013 & 4.783 & 0.033 \\
Time after surgery & 1.416 & 4 & 0.354 & 131.163 & 0.000 \\
Surgical methods - time after surgery & 0.049 & 4 & 0.012 & 4.4546 & 0.003 \\
\hline
\end{tabular}

df, degree of freedom; F, F test results; Sig., significance.

cell layers of the corneal epithelium, is one of the associated proteins expressed in the tight junctions that serve to link the junctions to actin filaments. The loss of ZO-1 may result in a disorganization of the tight junction, since it serves to organize the tight junction complex by recruiting membrane proteins, such as occludin and claudins, to the cell-cell contact sites, and also by connecting it to the actin cytoskeleton.

In our study, the pattern of claudin-1 and ZO-1 staining was used as the index of tight junction integrity and restoration after surgery in the corneal epithelium to determine the toxic effect of using $20 \%$ alcohol on corneal epithelial tight junctions during LASEK. We first tested the rate of epithelial wound closure after surgery. The timing of epithelial wound recovery was similar between the two groups where the rats had no apparent epithelial defect at approximately $48 \mathrm{~h}$. However, the immunfluorescence results showed that the expression of the tight junction proteins, claudin-1 and ZO-1, of the corneal epithelium treated with $20 \%$ alcohol was weaker and more faint compared to that of the cornea treated mechanically in the early post-operative period, and the speed of restoration of the alcohol-treated corneal epithelial tight junction was slower compared to the cornea treated mechanically. We also examined the mRNA levels of the two tight junction proteins in the central cornea after alcohol and mechanical treatment. The semi-quantitative RT-PCR analysis produced similar results.

The results from our study were in agreement with previous investigations. Kim et al (15) observed that one or two layers of superficial corneal epithelium showed morphological changes in the cytoplasm and surface damage, such as alterations in the microvilli structure and focal disruption of the intercellular junction, after treatment with $20 \%$ alcohol for $1 \mathrm{~min}$. The damage persisted until day five after alcohol treatment. Fernandez et al (16) determined that chronic alcohol ingestion affected the expression of multiple claudins; most striking were decreases in cladudin-1 and claudin-7 in the whole lung and in alveolar epithelial monolayers derived from alcohol-fed rats. This change increases lung epithelial permeability and promotes flooding of the alveolar airspaces with proteinaceous fluid in response to stresses, such as sepsis.

This examination showed that alcohol may have no significant effect on the epithelial wound closure, but was toxic to the corneal epithelial tight junction and delayed the restoration of the epithelial tight junction during the early post-operative period, especially at 24 and $48 \mathrm{~h}$ after surgery, although the wounded areas were recovered by the regenerated epithelium. The tight junction is the most important base during forming the epithelial barrier and also prevents fluid loss and the penetration of potential pathogens. An increase in permeability and the breakdown of epithelial barrier function are associated with corneal bacterial infections and loosening of tight junctions in the epithelium may contribute to destructive events during infection (8). The disruption of corneal epithelial barrier function is also a key clinical feature of keratitis sicca, which is a severe and sight-threatening complication of dry eyes (17). Therefore, we concluded that using $20 \%$ alcohol during LASEK might increase the risk of post-operative infection and inflammation, and the opportunity of complications; for example, dry eyes during the early post-operative period due to the toxic effect on corneal epithelial tight junctions. This result was partially consistent with the finding of previous clinical studies. Herrmann et al (18) reported that subjective symptoms of dye eyes presented during the first two months after LASEK. Horwath-Winter et al (19) observed that LASEK induced a short-term reduction in corneal sensation and slightly affected the ocular surface and tear film. Further research is required to reveal the role of corneal epithelial tight junctions in the complication of refractive surgery.

In conclusion, using $20 \%$ alcohol during LASEK has a toxic effect on the corneal epithelial tight junction and delays its restoration after surgery during the early post-operative period. Although the effects of alcohol do not continue for a long time (till $72 \mathrm{~h}$ ) after treatment and it has no significant effect on corneal epithelium wound closure, alcohol does have a possible contribution in increasing the risk of post- 
operative infection and complications. However, according to the previous studies about LASEK, it is still a safe surgical procedure with more rapid visual rehabilitation, less pain and less haze formation.

\section{Acknowledgements}

This study was supported by the Natural Science Foundation of Higher Educational Bureau of Anhui Province (KJ2009B088Z).

\section{References}

1. O'Keefe M and Kirwan C: Laser epithelial keratomileusis in 2010 - a review. Clin Experiment Ophthalmol 38: 183-191, 2010.

2. Azar DT, Ang RT, Lee JB, et al: Laser subepithelial keratomileusis: electron microscopy and visual outcomes of flap photorefractive keratectomy. Curr Opin Ophthalmol 12: 323-328, 2001.

3. Camellin M: Laser epithelial keratomileusis for myopia. J Refract Surg 19: 666-670, 2003.

4. Sugrue SP and JD Zieske: ZO1 in corneal epithelium: association to the zonula occludens and adherens junctions. Exp Eye Res 64: 11-20, 1997.

5. Wang Y,Zhang J, Yi XJ, et al: Activation of ERK1/2 MAP kinase pathway induces tight junction disruption in human corneal epithelial cells. Exp Eye Res 78: 125-136, 2004.

6. Tsukita S, Furuse M and Itoh M: Multifunctional strands in tight junctions. Nat Rev Mol Cell Biol 2: 285-293, 2001.

7. Furuse M, Furuse K, Sasaki H and Tsukita S: Conversion of zonulae occludentes from tight to leaky strand type by introducing claudin-2 into Madin-Darby canine kidney I cells. J Cell Biol 153: 263-272, 2001.

8. Yi X, Wang Y and Yu FS: Corneal epithelial tight junctions and their response to lipopolysaccharide challenge. Invest Ophthalmol Vis Sci 41: 4093-4100, 2000.
9. Michels RG and Maumenee AE: Retrobulbar alcohol injection in seeing eyes. Trans Am Acad Ophthalmol Otolaryngol 77: 164-167, 1973.

10. Campos M, Raman S, Lee M and McDonnell PJ: Keratocyte loss after different methods of de-epithelialization. Ophthalmology 101: 890-894, 1994.

11. Agrawal VB, Hanuch OE, Bassaqe S and Aquavella JV: Alcohol versus mechanical epithelial debridement: effect on underlying cornea before excimer laser surgery. J Cataract Refract Surg 23: 1153-1159, 1997.

12. Hutcheon AE, Sippel KC and JD Zieske: Examination of the restoration of epithelial barrier function following superficial keratectomy. Exp Eye Res 84: 32-38, 2007.

13. McCartney MD and Cantu-Crouch D: Rabbit corneal epithelial wound repair: tight junction reformation. Curr Eye Res 11: 15-24, 1992.

14. Wang Y, Chen M and Wolosin JM: ZO-1 in corneal epithelium; stratal distribution and synthesis induction by outer cell removal. Exp Eye Res 57: 283-292, 1993.

15. Kim SY, Sah WJ, Lim YW and Hahn TW: Twenty percent alcohol toxicity on rabbit corneal epithelial cells: electron microscopic study. Cornea 21: 388-392, 2002.

16. Fernandez AL, Koval M, Fan X and Guidot DM: Chronic alcohol ingestion alters claudin expression in the alveolar epithelium of rats. Alcohol 41: 371-379, 2007.

17. Pflugfelder SC,Farley W, Luo L, et al: Matrix metalloproteinase-9 knockout confers resistance to corneal epithelial barrier disruption in experimental dry eye. Am J Pathol 166: 61-71, 2005.

18. Herrmann WA, Shah CP, von Mohrenfels CW, et al: Tear film function and corneal sensation in the early postoperative period after LASEK for the correction of myopia. Graefes Arch Clin Exp Ophthalmol 243: 911-916, 2005.

19. Horwath-Winter J, Vidic B, Schwantzer G and Schmut O: Early changes in corneal sensation, ocular surface integrity, and tear-film function after laser-assisted subepithelial keratectomy. J Cataract Refract Surg 30: 2316-2321, 2004. 